\title{
DIAGNÓSTICO FÍSICO QUÍMICO DE LA CALIDAD DEL AGUA DE RIEGO EN EL CHACO HÚMEDO
}

\author{
Sosa, Olga M. ${ }^{12}$; Currie, Héctor M. ${ }^{1}$ Bartra Vásquez, Lady' ; Caseres, Silvia L. ${ }^{1}$ \\ ${ }^{1}$ Facultad de Ciencias Agrarias, UNNE. Sargento Cabral 2131 Corrientes, Capital. ${ }^{2}$ Dirección de Agricultura, Ministerio \\ de la Producción, Gobierno del Chaco. M.T de Alvear 145, $6^{\text {to }}$. Piso- Resistencia, Chaco. \\ marianasosa@agr.unne.edu.ar
}

\section{RESUMEN}

La calidad de las aguas para riego es determinada por la naturaleza, cantidad y proporción de iones presentes.Las sales solubles contenidas en el agua de riego producen efectos dañinos a las plantas al aumentar la concentración salina de la solución del suelo y el grado de saturación de sodio intercambiable. El concepto de Peligrosidad Salina está relacionado íntimamente al fenómeno del aumento de la succión osmótica de la solución del suelo y a la consiguiente disminución del potencial hídrico. Desde el punto de vista de la peligrosidad sódica del agua de riego, hay dos aspectos que interesan y son: el Porcentaje de Sodio Intercambiable (PSI), al que tenderá ese suelo con el agua de riego y la velocidad con que ese equilibrio se producirá. Con el propósito de establecer un diagnóstico de calidad de las aguas utilizadas para riego de hortalizas y frutales en el Chaco Húmedo, se han realizado análisis físico químico de muestras de agua de diferentes fuentes. Las aguas superficiales evaluadas resultaron aptas para riego en la totalidad de los casos, aunque el $6,46 \%$ de los casos deben utilizarse para riego con precauciones. El 55,56\% de las aguas subterráneas analizadas son aptas para riego.

Palabras clave: calidad de agua, riego, conductividad eléctrica, porcentaje de sodio intercambiable.

\footnotetext{
ABSTRACT

The quality of irrigation water is determined by nature, quantity and proportion of ions in the solution. Soluble salts present in irrigation water produce harmful effects on plants by increasing the salt concentration in soil solution, provoking the consequent decrease in water potential.There are two interesting aspects regarding the sodic danger of irrigation water: a) the Interchangeable Sodium Percentage (ISP), to which the soil tends with the use of irrigation water, and b) the speed at which this balance will occur. With the aim of testing water used for irrigation of vegetables and fruits in the Chaco Húmedo, physical and chemical analyses of water samples from different sources were carried out. Surface water assessed was suitable for irrigation use in all cases,
}

while $6.46 \%$ of the cases should be used for irrigation with precautions. Around $55.56 \%$ of the ground water tested was suitable for irrigation.

Key words: water quality, irrigation, electrical conductivity. Interchangeable Sodium Percentage (ISP)

\section{INTRODUCCIÓN}

Según las normas del Laboratorio de Salinidad de los Estados Unidos, las características más importantes que determinan la calidad de agua para riego son: la naturaleza, la cantidad y la proporción de los iones presentes en el agua. Las determinaciones fundamentales son (Handbook 60; citado por Avellaneda, 2004):

1. Concentración total de sales solubles.

2. Concentración relativa de sodio, respecto de otros cationes.

3. Concentración de boro u otros elementos que puedan ser tóxicos.

4. Bajo ciertas condiciones, la concentración de bicarbonatos con relación a la concentración de calcio más magnesio.

La composición química del agua es uno de los factores para establecer su aptitud de uso para riego, que está determinada por la concentración y composición de los constituyentes disueltos. El empleo de agua salina para riego altera las condiciones del suelo, afectando el crecimiento de las plantas.

Las sales solubles contenidas en el agua de riego producen efectos dañinos a las plantas al aumentar la concentración salina de la solución del suelo y el grado de saturación de sodio del complejo intercambiable. Este último es de efecto más permanente dado que generalmente persiste después de que las sales se han eliminado. También se deben mencionar los efectos tóxicos específicos de ciertos iones, tales como el boro, el litio, el sodio y el cloro, en concentraciones relativamente menores.

Kelley (1963), opina que al clasificar las aguas de riego deben considerarse como fundamentales los siguientes efectos: 
a. Los efectos sobre la succión osmótica (so) de la solución del suelo.

b. Los efectos sobre los cationes intercambiables del suelo, especialmente en relación con las propiedades físicas.

c. Los efectos sobre la relación de los cationes en la solución del suelo con respecto al crecimiento de las plantas.

En la mayoría de los cultivos, el crecimiento de las plantas está íntimamente relacionado con la succión osmótica en el medio que crecen las raíces, exhibiendo sensibilidades diferentes según las especies e incluso las variedades.

Las sales disueltas en el agua de riego por su parte desarrollan una succión osmótica y al incorporarse al suelo disminuyen la energía libre del suelo (succión osmótica + succión matriz). Por lo tanto el concepto de Peligrosidad Salina está ligado íntimamente al fenómeno de un aumento de la succión osmótica de la solución del suelo y a la consiguiente disminución del potencial hídrico.

El U.S. Salinity Laboratory (Riverside, California; citado por Avellaneda, 2004) en 1954 estableció una clasificación que combina el concepto de salinidad con el de sodicidad (Tabla 1).

Los constituyentes inorgánicos solubles de las aguas de riego reaccionan con los suelos en forma iónica. Los principales cationes son calcio, magnesio y sodio, con pequeñas cantidades de potasio. Los aniones principales son: carbonatos, bicarbonatos, sulfatos y cloruros, y en menor cantidad, nitratos, fluoruros y boratos.

Las sales disueltas en las aguas de riego pueden influenciar sensiblemente de las propiedades físicas de los suelos a través del intercambio catiónico. El principal peligro está representado por el sodio, ya que al aumentar su concentración en la solución edáfica, tiende a reemplazar al calcio y al magnesio del complejo coloidal, con la consecuente sodificación del suelo.

El uso continuo de un agua con una relativamente alta proporción de sodio respecto del calcio y del magnesio, puede eventualmente producir una elevada relación de sodio intercambiable y transformar el suelo en sódico.

El peligro de sodificación que entraña el uso de aguas de riego, queda determinado por las concentraciones absolutas y relativas de los cationes. La acción dispersante del sodio provoca la disgregación y dispersión del suelo, como consecuencia, éste tiende a adquirir una estructura física pobre, deficiente aeración, lenta infiltración, a veces $\mathrm{pH}$ elevado y representa un medio pobre para el crecimiento de las plantas.

Desde el punto de vista de la peligrosidad sódica de un agua de riego hay dos aspectos que interesan y
Tabla 1: Clasificación del U.S. Salinity Laboratory

\begin{tabular}{ccc}
\hline $\mathrm{CE}\left(\boldsymbol{\mu \mathrm { cm } ^ { - 1 }}\right.$ a $\left.\mathbf{2 5} \mathbf{0}\right)$ & Categoría & Calificación \\
\hline Hasta 250 & 1 & Salinidad baja \\
250 a 750 & 2 & Salinidad media \\
750 a 2250 & 3 & Salinidad alta \\
Mayor de 2250 & 4 & Salinidad muy alta \\
\hline
\end{tabular}

son: el Porcentaje de Sodio Intercambiable (PSI), al que tenderá ese suelo con el agua de riego y la velocidad con que ese equilibrio se producirá.

Para establecer el PSI de equilibrio el Laboratorio de Salinidad de Riverside propuso la determinación de la Relación de Adsorción de Sodio (RAS) sobre la base de las observaciones empíricas apoyadas teóricamente en la ley de acción de masas aplicadas al intercambio catiónico.

La importancia de la concentración total, en la velocidad de equilibrio, está reconocida en la clasificación de Riverside al asignarle mayor peligrosidad sódica, para una misma RAS, a medida que aumenta la conductividad eléctrica del agua, es decir su concentración salina. Este esquema divide las aguas por su peligrosidad sódica en cuatro clases:

S 1 - Baja peligrosidad sódica.

S 2 - Mediana peligrosidad sódica.

S 3 - Alta peligrosidad sódica.

S 4 - Muy alta peligrosidad sódica.

\section{ANTECEDENTES}

Como antecedentes en la región se halló el trabajo de Melgratti (2005) con el objeto de establecer la metodología para determinar la calidad de agua para riego y consumo animal.

En la Provincia de Corrientes, Rodríguez, et al. (2004) en Evaluación de la calidad del agua utilizada en el riego de arroz, en el Centro-Sur de CorrientesArgentina, valoraron la calidad de agua utilizada para riego en el cultivo de arroz provenientes de represas de tierra, y de ríos o arroyos.

Rodríguez et al. (2005) realizaron el análisis y clasificación de aguas utilizadas para riego en una arrocera del centro-sur de la Provincia de Corrientes, usando criterios del US Salinity Laboratory, 1951 (Riverside, citado en Avellaneda, 2004) y de la FAO (Ayers y Wescott, 1989 citados por Avellaneda, 2004), también clasificaron según el índice de Scott o coeficiente alcalinométrico (Cánovas, 1986; citado por Avellaneda 2004) y según su dureza expresada en grados hidrotimétricos Franceses, y concluyeron que en general, las muestras de agua analizadas no tendrían restricciones de uso.

Baccaro, K. et al. (2006) analizaron la calidad de aguas para consumo humano y para riego en el cinturón hortícola de Mar del Plata. Los análisis y la 
Tabla 2: Resultados de los análisis de aguas superficiales utilizadas para riego.

\begin{tabular}{|c|c|c|c|c|c|c|c|c|c|c|c|c|c|c|}
\hline 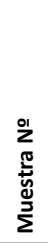 & & & $\frac{I}{2}$ & 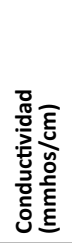 & 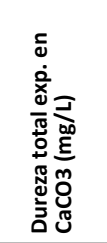 & 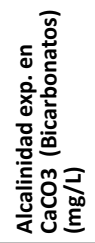 & 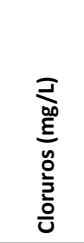 & 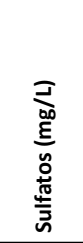 & 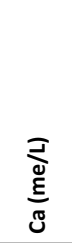 & $\frac{\Sigma}{\stackrel{\Sigma}{\Phi}}$ & 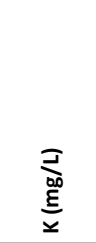 & 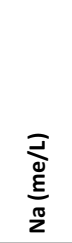 & $\stackrel{\tilde{x}}{\propto}$ & 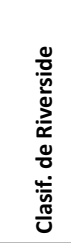 \\
\hline 1 & $26^{\circ} 26^{\prime} 52.75^{\prime \prime} \mathrm{S}$ & $59^{\circ} 18^{\prime} 39.76^{\prime \prime} \mathrm{W}$ & 4,6 & 255 & 66 & 45 & 17,50 & 5,28 & 0,44 & 0,87 & 50,31 & 0,90 & 1,11 & C2 S1 \\
\hline 2 & $26^{\circ} 28^{\prime} 41.19^{\prime \prime} \mathrm{S}$ & $59^{\circ} 17^{\prime} 44.04^{\prime \prime} \mathrm{W}$ & 6,2 & 355 & 70 & 78 & 27,00 & 33,57 & 1,00 & 0,39 & 57,73 & 1,60 & 1,92 & $\mathrm{C} 2 \mathrm{~S} 2$ \\
\hline 3 & $26^{\circ} 24^{\prime} 52.36^{\prime \prime} \mathrm{S}$ & $59^{\circ} 22^{\prime} 21.06^{\prime \prime} \mathrm{W}$ & 6,2 & 280 & 72 & 70 & 15,00 & 8,06 & 0,48 & 0,95 & 51,87 & 0,98 & 1,16 & C1 S1 \\
\hline 4 & $27^{\circ} 21^{\prime} 23.29^{\prime \prime} \mathrm{S}$ & $59^{\circ} 5^{\prime} 13.63^{\prime \prime} \mathrm{W}$ & 6,2 & 480 & 46 & 70 & 95,00 & 5,56 & 0,36 & 0,55 & 42,12 & 3,40 & 5,03 & C2 S1 \\
\hline 5 & $26^{\circ} 27^{\prime} 12.76^{\prime \prime} S$ & $59^{\circ} 18^{\prime} 48.33^{\prime \prime} \mathrm{W}$ & 6,6 & 250 & 60 & 70 & 22,50 & 11,08 & 0,40 & 0,79 & 43,68 & 0,86 & 1,12 & C1 S1 \\
\hline 6 & $26^{\circ} 2^{\prime} 1.75^{\prime \prime S}$ & $59^{\circ} 58^{\prime} 4.37^{\prime \prime} \mathrm{W}$ & 6,3 & 490 & 140,13 & 115 & 22,50 & 70,00 & 1,20 & 1,58 & 6,50 & 2,17 & 1,84 & C2 S1 \\
\hline 7 & $26^{\circ} 37^{\prime} 27.08^{\prime \prime S}$ & $59^{\circ} 48^{\prime} 11.45^{\prime \prime} \mathrm{W}$ & 6,5 & 170 & 62 & 55 & 7,50 & 17,30 & 0,80 & 0,43 & 46,02 & 0,40 & 0,51 & C1 S1 \\
\hline 8 & $27^{\circ} 21^{\prime} 13.90^{\prime \prime} \mathrm{S}$ & $58^{\circ} 57^{\prime} 38.52^{\prime \prime} \mathrm{W}$ & 5,9 & 260 & 128 & 185 & 27,60 & 19,58 & 0,92 & 62 & & 54 & 48 & C2 S1 \\
\hline 9 & $27^{\circ} 10^{\prime} 43.96^{\prime \prime} \mathrm{S}$ & $59^{\circ} 18^{\prime} 39.23^{\prime \prime} \mathrm{W}$ & 6,2 & 150 & 50 & 65 & 9,50 & 2,92 & 0,20 & 0,79 & 55,77 & 0,88 & 1,25 & $\mathrm{C} 1 \mathrm{~S} 1$ \\
\hline 10 & $26^{\circ} 27^{\prime} 19.76^{\prime \prime S}$ & $59^{\circ} 18^{\prime} 39.57^{\prime \prime} \mathrm{W}$ & 6,4 & 240 & 60 & 65 & 17,50 & 10,83 & 0,60 & 0,59 & 42,51 & 0,84 & 1,09 & C1 S1 \\
\hline 11 & $26^{\circ} 27^{\prime} 56.74^{\prime \prime} \mathrm{S}$ & $59^{\circ} 18^{\prime} 58.94^{\prime \prime} \mathrm{W}$ & 8,6 & 800 & 120 & 125 & 75,50 & 73,21 & 1,60 & 0,79 & 160,30 & 4,80 & 4,39 & C3 S1 \\
\hline 12 & $27^{\circ} 9^{\prime} 39.82^{\prime \prime S}$ & $59^{\circ} 20^{\prime} 5.44^{\prime \prime} \mathrm{W}$ & 6 & 90 & 200 & & & 15,24 & & & & & & $1 \mathrm{~S} 1$ \\
\hline 13 & $26^{\circ} 29^{\prime} 2.00^{\prime \prime} \mathrm{S}$ & $59^{\circ} 17^{\prime} 34.88^{\prime \prime} \mathrm{W}$ & 6,6 & 345 & 70 & 120 & 16,00 & 9,52 & 1,00 & 0,39 & 93,21 & 1,40 & 1,68 & C2 S1 \\
\hline 14 & $26^{\circ} 29^{\prime} 1.13^{\prime \prime S}$ & $59^{\circ} 17^{\prime} 35.41^{\prime \prime} \mathrm{W}$ & 5,6 & 80 & 31 & 42 & 8,00 & 31,67 & 0,46 & 0,16 & 10,14 & 0,52 & 0,94 & C1 S1 \\
\hline 15 & $26^{\circ} 33^{\prime} 45.14^{\prime \prime} \mathrm{S}$ & $59^{\circ} 17^{\prime} 56.98^{\prime \prime} \mathrm{W}$ & 7,5 & 150 & 40 & 57 & 10,50 & 17,31 & 0,50 & 0,30 & 11,70 & 5,20 & 8,24 & C1 S1 \\
\hline 16 & $26^{\circ} 33^{\prime} 42.46^{\prime \prime} \mathrm{S}$ & $59^{\circ} 18^{\prime} 24.77^{\prime \prime} \mathrm{W}$ & 6,1 & 165 & 25 & & 9,50 & 67 & $0,<4$ & & 3 & ,96 & 93 & C1 S1 \\
\hline 17 & $26^{\circ} 29^{\prime} 5.16^{\prime \prime} \mathrm{S}$ & $59^{\circ} 17^{\prime} 33.97^{\prime \prime} \mathrm{W}$ & 7 & 350 & 70 & 122 & 21,50 & 10,00 & & & 92,04 & 1,60 & 1,92 & C2 S1 \\
\hline 18 & $27^{\circ} 21^{\prime} 43.94^{\prime \prime} \mathrm{S}$ & $59^{\circ} 3^{\prime} 58.80^{\prime \prime} \mathrm{W}$ & 5,2 & 85 & 80 & 40 & 5,00 & 7,08 & 0,20 & 1,38 & 47,58 & 0,22 & 0,25 & C1 S1 \\
\hline 19 & $26^{\circ} 24^{\prime} 53.28^{\prime \prime} \mathrm{S}$ & $59^{\circ} 22^{\prime} 53.76^{\prime \prime} \mathrm{W}$ & 6,6 & 700 & 168 & 174 & 56,48 & 55,57 & 2,04 & 1,30 & 20,28 & 0,28 & 0,22 & C2 S1 \\
\hline 20 & $26^{\circ} 33^{\prime} 38.66^{\prime \prime} \mathrm{S}$ & $59^{\circ} 17^{\prime} 36.24^{\prime \prime} \mathrm{W}$ & 6,9 & 700 & 108 & 73 & 62,00 & 72,50 & 1,22 & 93 & 0,09 & 2,40 & 2,32 & C2 S1 \\
\hline 21 & $26^{\circ} 33^{\prime} 34.42^{\prime \prime} \mathrm{S}$ & $59^{\circ} 17^{\prime} 36.24^{\prime \prime} \mathrm{W}$ & 6,6 & 450 & 103 & 127 & 34,00 & 47,10 & 1,20 & 0,85 & 25,35 & 2,40 & 2,37 & C2 S1 \\
\hline 22 & $26^{\circ} 6^{\prime} 31.93^{\prime \prime S}$ & $59^{\circ} 56^{\prime} 0.60^{\prime \prime} \mathrm{W}$ & 6,5 & 500 & 140,13 & 123 & 20,00 & 57,69 & 1,20 & 1,58 & & 2,23 & 1,89 & C2 S1 \\
\hline 23 & $26^{\circ} 38^{\prime} 49.86^{\prime \prime} \mathrm{S}$ & $59^{\circ} 19^{\prime} 36.63^{\prime \prime} \mathrm{W}$ & 6,6 & 400 & 78 & 83 & 39,50 & 22,50 & 0,92 & 0,63 & 30,42 & 1,60 & 1,82 & C2 S1 \\
\hline 24 & $26^{\circ} 26^{\prime} 46.10^{\prime \prime} \mathrm{S}$ & $59^{\circ} 19^{\prime} 13.96^{\prime \prime} \mathrm{W}$ & 7,2 & 700 & 191 & 111 & 33,50 & 67,16 & 2,36 & 1,44 & 32,76 & 2,40 & 1,74 & C2 S1 \\
\hline 25 & $26^{\circ} 26^{\prime} 51.55^{\prime \prime} \mathrm{s}$ & $59^{\circ} 19^{\prime} 2.27^{\prime \prime} \mathrm{W}$ & 6 & 380 & 84 & 88 & 30,50 & 21,67 & 0,90 & 0,77 & 18,72 & 0,14 & 0,15 & C2 S1 \\
\hline 26 & $26^{\circ} 26^{\prime} 49.17^{\prime \prime} \mathrm{S}$ & $59^{\circ} 19^{\prime} 6.37^{\prime \prime} \mathrm{W}$ & 6,6 & 400 & 84 & 86 & 31,50 & 25,23 & 1,10 & 0,57 & 17,16 & 1,20 & 1,31 & C2 S1 \\
\hline 27 & $26^{\circ} 10^{\prime} 38.46^{\prime \prime} \mathrm{S}$ & $59^{\circ} 34^{\prime} 35.00^{\prime \prime} \mathrm{W}$ & 5,6 & 500 & 90,08 & 162 & 39,50 & 16,92 & 1,00 & 0,79 & 27,00 & 2,46 & 2,60 & C2 S1 \\
\hline 28 & $26^{\circ} 24^{\prime} 55.69^{\prime \prime} \mathrm{S}$ & $59^{\circ} 22^{\prime} 54.80^{\prime \prime} \mathrm{W}$ & 6,4 & 600 & 146 & 129 & 59,00 & 67,16 & 1,84 & 1,07 & 14,43 & 2,60 & 2,16 & C2 S1 \\
\hline 29 & $26^{\circ} 41^{\prime} 31.20^{\prime \prime} \mathrm{S}$ & $59^{\circ} 8^{\prime} 39.28^{\prime \prime} \mathrm{W}$ & 6,1 & 230 & 58 & 100 & 7,50 & 8,89 & 0,60 & 0,55 & 32,76 & 1,12 & 1,48 & C1 S1 \\
\hline 30 & $26^{\circ} 8^{\prime} 22.74^{\prime \prime S}$ & $59^{\circ} 35^{\prime} 31.52^{\prime \prime} \mathrm{W}$ & 6,8 & 350 & 120,11 & 89 & 18,00 & 85,77 & 1,80 & 0,59 & 3,90 & 1,82 & 1,67 & $\mathrm{C} 2 \mathrm{~S} 1$ \\
\hline
\end{tabular}

clasificación de las aguas de riego fueron realizados según las Normas de Riverside del Laboratorio de Salinidad de Estados Unidos. Han hallado que el alto contenido de sales determina que dichas aguas no sean aptas para riego, especialmente en suelos con drenaje limitado.

\section{OBJETIVO}

Establecer un diagnóstico de calidad de las aguas utilizadas para riego de hortalizas y frutales en el Chaco Húmedo.

\section{MATERIALES Y MÉTODOS}

Se tomaron muestras de agua de distintas fuentes, en predios con equipo de bombeo instalado. Para realizar el muestreo se dejó sin funcionar el equipo de bombeo durante cinco minutos, luego se enjuagó tres veces el recipiente y se tomó la muestra de 1,5 litros de agua.

Las fuentes de agua fueron las siguientes:

- Agua de pozo

- Agua de perforación

- Agua de Río de Oro.

- Agua de Río Guaycurú

Se determinó $\mathrm{pH}$ por Potenciometría, conductividad eléctrica (CE): por Conductimetría (cónductímetro estandarizado a $25^{\circ} \mathrm{C}$ ), alcalinidad total: por volumetría de neutralización (APHA, 1989), dureza total: por Complexometría, Sodio y Potasio: por Espectrometría de Absorción Atómica (APHA, 1989), Calcio y Magnesio: por volumetría de formación de complejos (APHA, 1989), cloruros: por Volumetría de Precipitación, método de Mohr (APHA, 1989), sulfatos: por Espectrometría de Absorción Molecu- 
lar-Turbidimetría (Metodo ASTM D 516-90) (Annual book of Standards 1994).

\section{RESULTADOS Y DISCUSIÓN}

Los resultados de los análisis de las aguas muestreadas se resumen en las Tablas $\mathrm{N}^{\circ} 2$ y $\mathrm{N}^{\circ} 3$ correspondientes a aguas superficiales y subterráneas respectivamente. Según los resultados expuestos en las Tablas 2 y 3 de los análisis de agua cuyas muestras fueron tomadas en la Provincia del Chaco, tanto las aguas superficiales como las subterráneas presentan aptitud para riego.

Las aguas superficiales resultan aptas para el riego, en un $35,5 \%$ de los casos evaluados, podría ser necesario emplear volúmenes de agua en exceso para lavar el suelo y utilizar cultivos tolerantes a la salinidad. No presentan limitantes con respecto a los niveles de sodicidad en un $96,8 \%$, representados en el gráfico $\mathrm{N}^{\mathrm{o}} 1$.

La conductividad eléctrica categoriza las aguas superficiales como aguas de salinidad media y baja. En cuanto a la peligrosidad sódica, sólo un caso de mediana peligrosidad y las restantes muestras de agua superficiales resultaron de baja peligrosidad sódica.

En cambio en las aguas subterráneas, según la conductividad eléctrica son categorizadas como de media y alta salinidad; y se identificaron dos casos de de mediana peligrosidad sódica, las restantes resultaron de baja peligrosidad.

Las aguas de perforaciones que han sido material de análisis para el presente trabajo, son aptas para riego teniendo en cuenta tanto el contenido de sales totales así como su Relación de Adsorción de Sodio (RAS).

En el gráfico $\mathrm{N}^{\circ} 1$ se observa que el $56 \%$ de las aguas subterráneas muestreadas son de salinidad media y el $44 \%$.

El gráfico $\mathrm{N}^{\circ} 2$ representa las frecuencias de calidad de agua. Se observa que la clase C2S1 es la de mayor frecuencia con una frecuencia de 0,57 para aguas superficiales y de 0,55 para aguas subterráneas, seguida por la clase C1S1 en aguas superficiales con

Tabla 3: Resultados de análisis de aguas subterráneas utilizadas para riego.

\begin{tabular}{|c|c|c|c|c|c|c|c|c|c|c|c|c|c|c|}
\hline 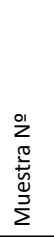 & $\begin{array}{l}\text { Corder } \\
\text { Geogr }\end{array}$ & $\begin{array}{l}\text { nadas } \\
\text { áficas }\end{array}$ & $\frac{I}{2}$ & 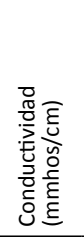 & 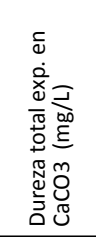 & 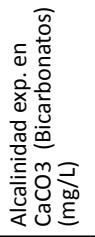 & 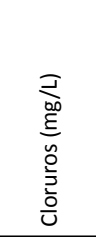 & 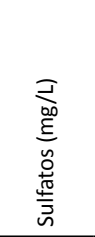 & 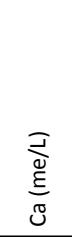 & 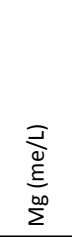 & 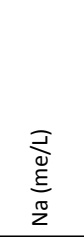 & 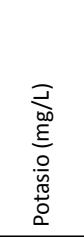 & $\frac{u}{\alpha}$ & 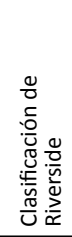 \\
\hline 1 & $27^{\circ} 16^{\prime} 34.96^{\prime \prime} \mathrm{S}$ & $59^{\circ} 0^{\prime} 16.33^{\prime \prime} \mathrm{W}$ & 6,6 & 440 & 184 & 155 & 19,00 & 17,08 & 1,92 & 1,74 & 0,72 & 20,28 & 0,53 & C2 S1 \\
\hline 2 & $27^{\circ} 58^{\prime} 20.57^{\prime \prime S}$ & $59^{\circ} 13^{\prime} 27.18^{\prime \prime} \mathrm{W}$ & 6,8 & 1200 & 193 & 289 & 7,50 & 701,09 & 5,20 & 2,49 & 5,20 & 8,97 & 2,65 & C3 S1 \\
\hline 3 & $27^{\circ} 14^{\prime} 44.21^{\prime \prime S}$ & $59^{\circ} 12^{\prime} 48.83^{\prime \prime} \mathrm{W}$ & 7,3 & 900 & 190 & 360 & 9,00 & 90,71 & 1,20 & 2,57 & 5,40 & 30,81 & 3,94 & C3 S1 \\
\hline 4 & $27^{\circ} 29^{\prime} 8.99^{\prime \prime} \mathrm{S}$ & $60^{\circ} 53^{\prime} 23.50^{\prime \prime} \mathrm{W}$ & 6 & 1650 & 730,66 & 314 & 108,50 & 365,38 & 4,10 & 1,04 & 10,13 & 31,00 & 6,32 & C3 S2 \\
\hline 5 & $27^{\circ} 10^{\prime} 2.77^{\prime \prime S}$ & $59^{\circ} 19^{\prime} 53.04^{\prime \prime} \mathrm{W}$ & 6,8 & 700 & 150 & 303 & 22,50 & 24,64 & 0,60 & 2,17 & 2,40 & 10,35 & 2,04 & $\mathrm{C} 2 \mathrm{~S} 1$ \\
\hline 6 & $26^{\circ} 27^{\prime} 57.15^{\prime \prime S}$ & $59^{\circ} 16^{\prime} 21.37^{\prime \prime} \mathrm{W}$ & 6,9 & 600 & 220,2 & 240 & 6,00 & 11,43 & 2,60 & 1,78 & 2,40 & 43,68 & 1,62 & $\mathrm{C} 2 \mathrm{~S} 1$ \\
\hline 7 & $27^{\circ} 16^{\prime} 53.94^{\prime \prime S} \mathrm{~S}$ & $58^{\circ} 59^{\prime} 25.37^{\prime \prime} \mathrm{W}$ & 6,8 & 550 & 244 & 200 & 5,80 & 74,31 & 2,68 & 2,17 & 1,08 & 24,57 & 0,69 & $\mathrm{C} 2 \mathrm{~S} 1$ \\
\hline 8 & $26^{\circ} 51^{\prime} 23.36^{\prime \prime} \mathrm{S}$ & $59^{\circ} 48^{\prime} 33.70^{\prime \prime} \mathrm{W}$ & 7,4 & 800 & 248 & 220 & 60,00 & 25,41 & 3,88 & 1,07 & 2,60 & 51,48 & 1,65 & C3 S1 \\
\hline 9 & $27^{\circ} 17^{\prime} 30.66^{\prime \prime} \mathrm{S}$ & $58^{\circ} 58^{\prime} 33.89^{\prime \prime} \mathrm{W}$ & 6,2 & 450 & 192 & 180 & 1,50 & 17,08 & 2,32 & 1,50 & 0,56 & 24,18 & 0,41 & C2 S1 \\
\hline 10 & $26^{\circ} 33^{\prime} 18.65^{\prime \prime} \mathrm{S}$ & $59^{\circ} 48^{\prime} 53.57^{\prime \prime} \mathrm{W}$ & 7,1 & 850 & 330 & 375 & 17,50 & 55,95 & 4,52 & 2,02 & 2,40 & 55,77 & 1,33 & C3 S1 \\
\hline 11 & $27^{\circ} 0^{\prime} 15.3^{\prime \prime S}$ & $60^{\circ} 53^{\prime} 07.4^{\prime \prime} \mathrm{W}$ & 7,4 & 1650 & 602,54 & 195 & 101,50 & 550,00 & 3,03 & 8,92 & 10,71 & 27,00 & 4,38 & C3 S2 \\
\hline
\end{tabular}

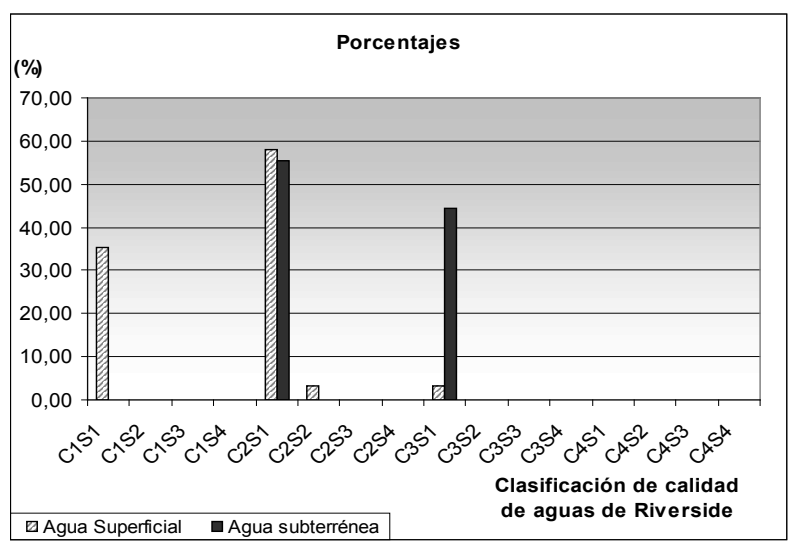

Gráfico No 1: Porcentajes por Clases de Aguas.

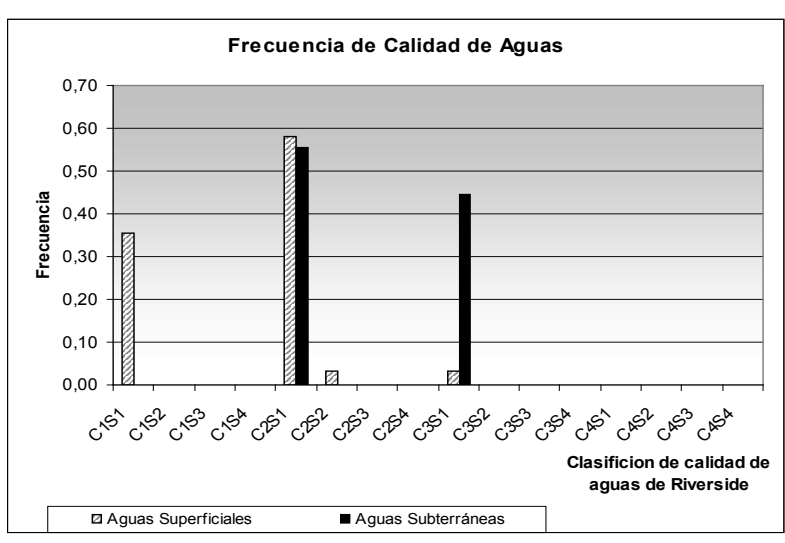

Gráfico No 2: Frecuencias de Clases de Aguas. 
una frecuencia de 0,36; C2S2 cuya frecuencia es de 0,5 y C3S1 con una frecuencia de 0,5 . En el caso de las aguas subterráneas la menor frecuencia la presenta la Clase C3S1.

\section{CONCLUSIONES}

El 93,54\% de muestras de agua superficial y el $55,56 \%$ de las aguas subterráneas son aguas de buena calidad, aptas para el riego. El 6,46\% de las aguas superficiales y el $44,44 \%$ de las aguas subterráneas son utilizables para el riego con precauciones.

\section{BIBLIOGRAFÍA}

American Society For Testing And Materials. Annual Book Of Standars. 1994. Determinación de sulfatos por turbidimetría en agua.

American Public Health Association (APHA). American Water Works Association and Water Pollution Control Federation. 1989. Standard Methods for the Examination OF Water and Wastewater, $17^{\text {th }}$ ed., Washington, D.C. USA. Parte 9000.
Avellaneda, M.O., Bermejillo, A.I., Mastrantonio, L.E. 2004. Aguas de riego. Calidad y evaluación de su factibilidad de uso.

Baccaro, K.; Degorgue, M.; Lucca, M.; Picone, L.; Zamuner, E.; Andreoli, Y. 2006.Calidad de Agua Para Consumo Humano y Para Riego en Muestras del Cinturón Hortícola de Mar del Plata. RIA, 35 (3): 95-110. INTA, Argentina.

Melgratti De Inalbon, M.R. Procedimientos Analíticos Para Aguas De Riego Y Consumo Animal, realizado en la EEA - INTA Pcia Roque Saenz Peña.

Rodriguez, S.C., Martinez, G.C.; Currie, H.M. 2004. Evaluación de la Calidad de Agua de Riego utilizada en el cultivo de arroz. Universidad Nacional del Nordeste, Jornadas de Comunicaciones Científicas y Tecnológicas

Rodriguez, S.C., Vara, J.; Martinez, G.C. 2005. Clasificación de las Aguas Utilizadas Para Riego en una Arrocera del Centro-Sur de Corrientes. Universidad Nacional del Nordeste, Jornadas de Comunicaciones Científicas y Tecnológicas. 